\title{
The Psychological Experience and Intervention in Post-Acute COVID-19 Inpatients
}

This article was published in the following Dove Press journal:

Neuropsychiatric Disease and Treatment

\author{
Silvia Rossi Ferrario (D) ${ }^{1, *}$ \\ Anna Panzeri $\mathbb{D}^{1,2 *}$ \\ Paola Cerutti (iD) \\ Daniela Sacco (ID) \\ 'Psychology and Neuropsychology Unit, \\ Institute of Veruno, Istituti Clinici \\ Scientifici Maugeri IRCCS, Veruno, Italy; \\ ${ }^{2}$ Department of General Psychology, \\ University of Padova, Padova, Italy \\ *These authors contributed equally to \\ this work
}

Correspondence: Anna Panzeri

Department of General Psychology,

University of Padova, Padova via Venezia 8,

Padova, 35131, Italy

Email anna.panzeri@phd.unipd.it
Purpose: COVID-19 patients faced first-hand the life-threatening consequences of the disease, oftentimes involving prolonged hospitalization in isolation from family and friends. This study aimed at describing the psychological intervention to address the psychological difficulties and issues encountered by the hospitalized post-acute COVID-19 patients in a rehabilitation setting.

Patients and Methods: Patients' demographics, medical diagnosis, and neuropsychological information were collected from March $2^{\text {nd }}$ to May $12^{\text {th }}, 2020$. The main psychological issues and intervention strategies were collected.

Results: A total of 181 patients were hospitalized during this period. Among them, the $47.5 \%$ underwent psychological assessment ( $\mathrm{N}=86$; age: $74.58 \pm 13.39 ; 54.7 \%$ females). The most common psychological issues were acute stress disorders (18.6\%), anxious and demoralization symptoms $(26.7 \%)$, depression $(10.5 \% \%)$, and troublesome grief $(8.1 \%)$. Once recovered from COVID-19, many patients were discharged home $(38.4 \%)$, some received further rehabilitation in non-COVID-19 wards (41.9\%), mostly due to pre-existent diseases (72.2\%) rather than to COVID-19 complications (27.8\%).

Conclusion: A great number of the hospitalized post-acute COVID-19 patients showed psychological issues requiring psychological intervention, the most common were anxiety, demoralization, acute stress, depression, and grief. The proposed psychological treatment for hospitalized COVID-19 patients was conducted in a Cognitive Behavioral framework. In particular, during the COVID-19 pandemic, psychological intervention is an important part of rehabilitation in the post-acute phase of the illness to reduce distress symptoms and improve psychological health.

Keywords: psychological intervention, COVID-19 patients, clinical psychology, health psychology, rehabilitation

\section{Introduction}

The novel beta-coronavirus SARS-CoV-2 (COVID-19), discovered in December 2019, is responsible for a severe systemic multi-organ disease. The disease is primarily characterized by a respiratory infection, but it can also involve multiple-organ impairment, with chronic and, possibly, lethal consequences. ${ }^{1}$

The ease with which the virus is transmitted from one person to another facilitated the worldwide spread of COVID-19, causing the ongoing pandemic emergency. ${ }^{2}$ These events have had devastating consequences on people's health, as well as on society and the economy.

Alongside China and other Asian countries, Italy was among the first countries worldwide, and the first in Europe, dealing with the effects of the COVID-19 
pandemic. In order to control the spread of the virus, the Italian government ordered a national lockdown, which lasted roughly 2 months, from $8^{\text {th }}$ March to $18^{\text {th }}$ May. The total number of deaths in this period reached almost 30.000 and the total number of infected cases was above $221.000 .^{3}$ Because the healthcare system could not provide enough swabs for every infected person, the number of infected people might have been much higher.

The precautionary measures adopted during the lockdown period were very restrictive and consisted of social distancing (from family members as well, if necessary), school closures, reduction or suspension of business activities, and smart working, with remarkable economic costs. ${ }^{4}$

Research has shown that feelings of fear, anxiety, uncertainty, loneliness, and depression were common in people forced to stay at home, independently from their COVID-19 diagnosis. ${ }^{5-7}$ Interestingly, some Chinese authors had already stressed that the psychological burden related to COVID-19 frequently occurred. ${ }^{8}$ It has been suggested that such complications should be considered as a first-order issue to be addressed through psychological and social intervention, ${ }^{9-11}$ which might involve webbased technologies. $^{12}$

It must be noted that the risk of developing psychological issues is higher for people with previous psychological and/or psychiatric issues, children with developmental disorders or learning difficulties, people subjected to domestic violence, and frail elders. ${ }^{13-15}$

The Italian government drafted specific guidelines to provide the right psychological intervention for infected patients and their families, as well as for the population as a whole. ${ }^{16}$ In this context, specific web-based technologies were used, such as PCs, tablets, and smartphones, allowing online video calls (eg, Skype, Zoom). ${ }^{17-19}$

It must be mentioned that no data was available over the psychological assessment and intervention which hospitalized acute COVID-19 and post-acute COVID-19 patients underwent. In many places, such as our Institute, post-acute COVID-19 patients (from here called COVID19 patients) were admitted to a dedicated department to allow the continuation of necessary treatments and to provide rehabilitation.

Furthermore, to prevent the COVID-19 diffusion, caregivers (defined as the most important people in the patient's life $)^{20}$ were denied to visit the patient. The absence of social support from family and friends represented an additional source of stress ${ }^{21}$ for both patients and caregivers, who were constantly worried about each other health conditions.

Thus, the COVID-19 disease and the related pandemic represent a consistent stressor for patients with COVID19, who showed particular clinical issues and specific needs. For this reason, the psychological intervention that was already an important part of rehabilitation $\operatorname{programs}^{22,23}$ - had to be reconsidered and re-adapted to the current situation.

The present study intends to explore the most common psychological experiences and difficulties of post-acute COVID-19 patients and to describe the procedure and techniques of the psychological intervention that was conducted.

\section{Patients and Methods Participants}

The study focused on patients with COVID-19 hospitalized in a post-acute rehabilitation Institute in Northern Italy during the outbreak. A convenience sampling was used, in particular, patients were progressively enrolled in the study as they were admitted to the COVID-19 ward. The sample included a total of 181 patients with a mean age of $75.27 \pm 12.45$ (53\% females, $n=96)$. The inclusion criteria were: being admitted in the COVID-19 ward of the hospital from $2^{\text {nd }}$ March to $12^{\text {th }}$ May 2020; having at least 18 years old; and good use of the Italian language. The exclusion criteria were: the absence of severe physical, cognitive, language issues preventing from conducting the psychological intervention and/or refusing to receive it.

\section{Setting and Procedure}

Patients with COVID-19 were hospitalized in a dedicated post-acute COVID-19 department on the upper floor of the building - according to the health and scientific direction of the Institute. Patients were treated for the comorbidities and the complications of the disease. They were provided physical rehabilitation treatments in their rooms or a dedicated space in the ward while waiting 24 hours for the two COVID-19 swabs results. If needed, rehabilitation was continued in non-COVID-19 departments once the infection was overcome.

The professional team included physicians with various specialties: physicians, nurses, health-care assistants, physiotherapists, and psychologists. The staff was already employed at the Institute or was hired for the pandemic period. Every member of the team wore the COVID-19 
protective equipment: a suit, gloves, a visor, and a mask. Separated entrance and exit paths were designed to avoid unnecessary contact.

As a routine procedure at the Institute, psychologists were notified about the need for psychological intervention by the other members of the team (eg, the physician) or by patients spontaneously asking for help. Thus, only a subset of all patients hospitalized in the COVID-19 ward was assessed and treated by psychologists. If a patient was successively transferred to a non-COVID-19 department in the same Institute, the psychological intervention was continued.

Some dedicated personnel with great communication skills daily reported the patients' clinical condition to their principal caregivers, who were not allowed to access the Institute.

\section{Psychological Intervention}

Two psychologists and psychotherapists (Authors SRF and PC), specialized in Cognitive Behavioral Therapy (CBT) and with a neuropsychological background, conducted the psychological intervention in all its phases with shifts in the COVID-19 department lasting six hours each, four afternoons a week.

As a first step, psychologists visited the patients in their rooms to assess their psychological and cognitive conditions. The interdiction of paper-pen assessment methods, together with the lack of computerized tools, contributed to the difficulties faced in the new situation of the COVID19 viral emergency. For these reasons, the psychologists chose to privilege the clinical interview to conduct the clinical assessment and to emphasize the patients' thoughts, emotions, and psychological issues. The clinical interview allowed to overcome the relational barriers generated by protective clothes, thus strengthening the use of paraverbal and non-verbal communication (in particular, cadence/tone of voice and gestures) as mediators for empathy. Unfavorable environmental conditions (eg: hygienic reasons and lack of dedicated space with privacy and silence) did not allow the use of any neuropsychological tool.

For every examined patient, the psychologists produced an electronic written report describing the patients' conditions, as well as stating the need (or not) for psychological treatment and the possible psychological diagnosis and clinical issues.
The patients' caregivers were contacted by telephone to gather information about the patients' life and past emotional experiences.

\section{Psychological Techniques}

The intervention was conducted within a CBT framework. In order to adapt the intervention to each patient's needs, no standardized procedures were followed. The following techniques were used and tailored to individual clinical conditions.

Given the age of the sample, the psychological treatment for elderly patients was tailored to their specific needs both given the age and the physical issue they are dealing with. As standard treatment in a rehabilitation context, the most important points of the intervention were: understanding and accepting own's psychophysical condition and/or illness; ${ }^{24}$ elaborating the psychological (emotional) reactions to it, such as anxiety, depression, as well as illness denial and conscious avoidance of the illness $;{ }^{25}$ reinforcing the motivation and adherence to rehabilitation treatments; ${ }^{26}$ reinforcing healthy habits that can be maintained after discharge (eg, physical exercise, weight management); ${ }^{27,28}$ favoring the adaptation to new life conditions; preparation to discharge to home and adaptation to daily life. Beyond these "standard" points, some patients may need specific intervention because of particular conditions. As an instance, some require personalized neuropsychological rehabilitation after stroke, head trauma, and neurosurgeries. Others may require a less intense neuropsychological treatment aimed to maintain or stimulate the (residual) cognitive resources. Some patients show particular psychological reactions such as anxiety, depression that can require more structured CBT protocols in order to reduce the symptomatology. Sometimes relational issues with relatives require conducting meetings with the family to favor the communication and to understand the patient psychophysical conditions and their consequences on daily life. Another issue related to older age is loneliness, often elders have to learn coping with feelings of solitude and bereavement. ${ }^{29,30}$

Given that the COVID-19 pandemic posed new challenges for patients, the psychological intervention was readapted to target also the COVID-19 related issues. Indeed, the pandemic spread fear, worries, and uncertainty about their own and the relatives' health. Furthermore, patients with COVID-19 and their caregivers were exposed both to self and others' sufferance because of COVID-19, some of them lived traumatic experiences or 
had losses because of the disease. Moreover, patients and caregivers were not allowed to meet face to face.

As a first step of the COVID-related psychological intervention, educative interventions provided patients (and caregivers during the calls) updated and realistic information about the COVID-19 disease.

A narrative approach was used to help patients with recalling and re-ordering their experiences, from the diagnosis of COVID-19 to the admission to the rehabilitation department. The process allowed for the identification of the subjective meaning of the various experiences' frames, ${ }^{31}$ as well as for the selection of the best strategy to apply.

Individual relaxation techniques, such as muscular relaxation, body-scan, breath control, and imaginative relaxation were performed on patients showing anxiety and acute stress symptoms. ${ }^{32}$ The cognitive restructuring was used to dispute and modify the irrational thoughts and maladaptive interpretations. ${ }^{33}$ The grounding techniques, mental as well as physical, were also used to manage intense negative emotions (eg, anxiety, sadness). ${ }^{34}$

In addition to this, psychologists conducted video calls through a dedicated tablet device to allow patients without a smartphone or not able to use it to keep contact with caregivers at distance, thus facilitating the communication between them and enhancing their relationship quality. ${ }^{35}$ This allowed to relief the feelings of loneliness related to the prolonged hospitalization and isolation. Moreover, during the video calls between patients and caregivers it was possible to correct some reciprocal misinterpretations and to observe any difficulties in the relationship.

\section{Variables}

The following variables about patients were collected: demographics (sex, age); medical diagnosis and comorbidities; medical outcome; undergoing psychological assessment (yes/no); psychological-neuropsychological issues; video calls with caregivers (yes/no); psychological support for the principal caregiver (yes/no).

\section{Analysis}

Descriptive statistics were used to provide an overview of the patients' characteristics. The most relevant medical and psychological conditions were reported with frequencies and percentages. The $t$-test and chi-square $\left(\chi^{2}\right)$ analysis were employed to compare, respectively, the patients' age and psychological issues across males and females. The effect sizes were calculated with Cohen's $d$. The alpha level was set at 0.05 . The R software was used. ${ }^{36}$

\section{Ethics Statements}

The study was authorized and approved by the Scientific Direction of the Maugeri Clinical and Scientific Institute of Veruno - where the study was conducted. The present study describes the clinical practice of the Institute during the COVID-19 emergency, no other procedure different than ordinary clinical practice was used. All the study participants willingly provided informed consent to use their data for research purposes. The guidelines outlined in the Declaration of Helsinki were followed. ${ }^{37}$

\section{Results}

From $2^{\text {nd }}$ April up to $12^{\text {th }}$ May 2020, a total of 181 patients with COVID-19 (mean age $=75.27 \pm 12.45$ ) were admitted to the post-acute COVID-19 ward (53\% females, $\mathrm{n}=96)$. Females were older (mean 78.21 \pm 12.13 ) than males (mean $71.94 \pm 12.04)$ with a significant difference $\left(\mathrm{t}_{(179)}=3.48, d=\right.$ $0.52 ; p<0.001)$. Figure 1 shows the study flow diagram.

This study focused on the subset that underwent psychological assessment, namely the $47.5 \%$ of the sample (n $=86$; mean age $74.58 \pm 13.39 ; 54.7 \%$ females). Also in this subset, females (mean 78.21 \pm 12.28 ) were older than males (mean $70.21 \pm 13.51$ ) with a statistically significant difference $\left(\mathrm{t}_{(84)}=2.88, d=0.84, p<0.005\right)$.

Of these, in $70.9 \%,(n=61)$ the reported medical diagnosis was only related to the novel COVID-19 disease, whilst the remaining patients $(29.1 \%)$ also showed already pre-existing medical conditions (Figure 2). No significant differences emerged between males and females $\left(\chi_{(1)}^{2}=0.629, p=0.428\right)$.

After psychological assessment and a dedicated psychoeducational session about the COVID-19, only $6.9 \%(n=6)$ of the patients showed good adaptation and any important psychological issue emerged, thus not requiring more structured psychological treatment. Moreover, $11.6 \%(\mathrm{n}=10)$ of patients were principally supported only with video-calls to favor the communication and the relationship with caregivers.

However, the majority of patients $(n=65,75.6 \%)$ displayed more severe (neuro)psychological difficulties and required further and structured psychological treatment. There were no significant differences across $\operatorname{sex}\left(\chi_{(1)}^{2}=\right.$ $0.554, p=0.457)$.

Among patients who underwent psychological assessment $(\mathrm{n}=86)$, the patients needing structured psychological treatment displayed various psychological/neuropsychological issues and diagnoses (each patient could have more than one): already known cognitive impairment $(\mathrm{n}=18,20.9 \%)$, 


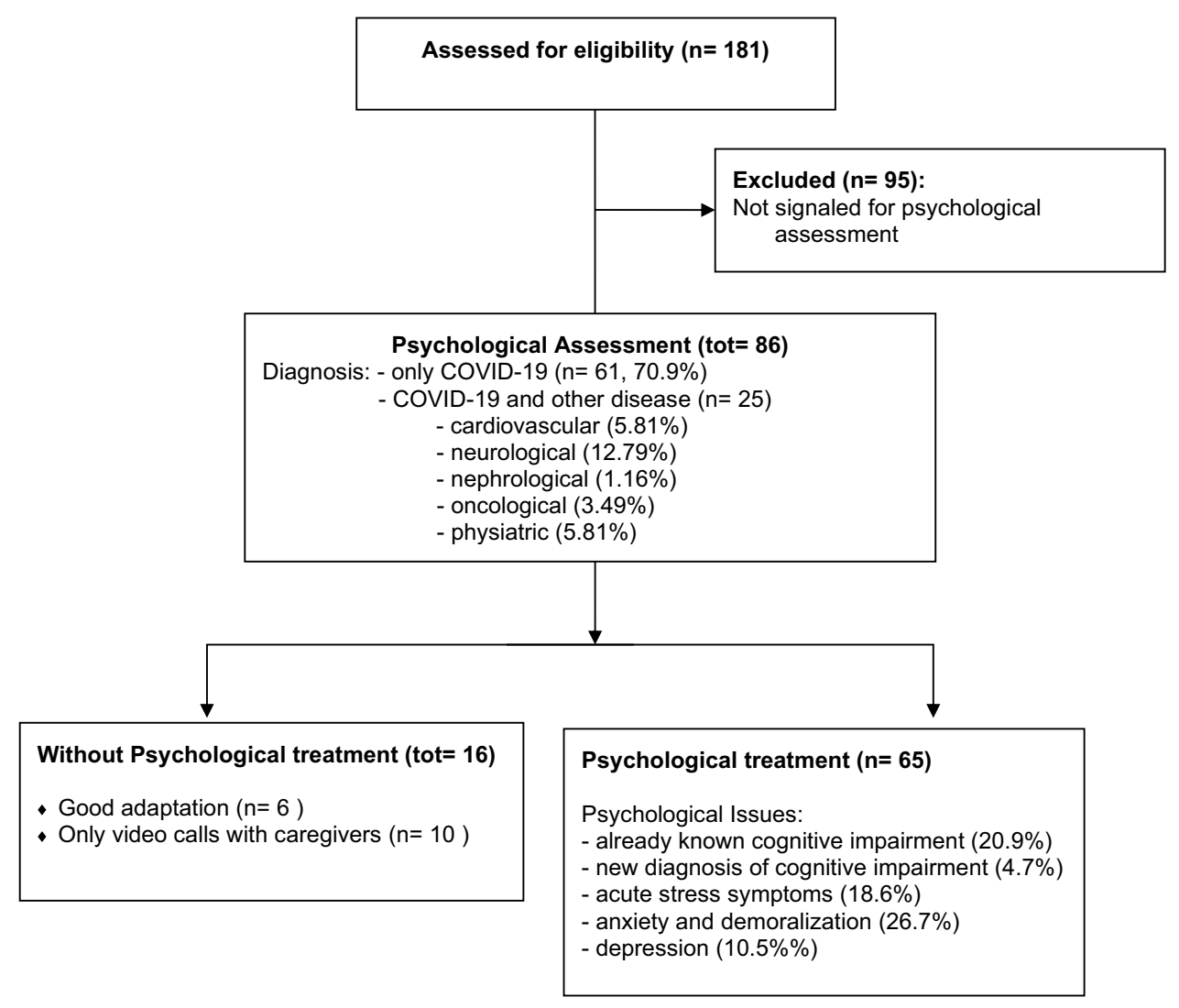

Figure I Study flowchart of participants and their characteristics.

Notes: This flowchart describes the flow of the sample participants from enrollment up to psychological intervention. The prevalence of the psychological diagnoses is also reported.

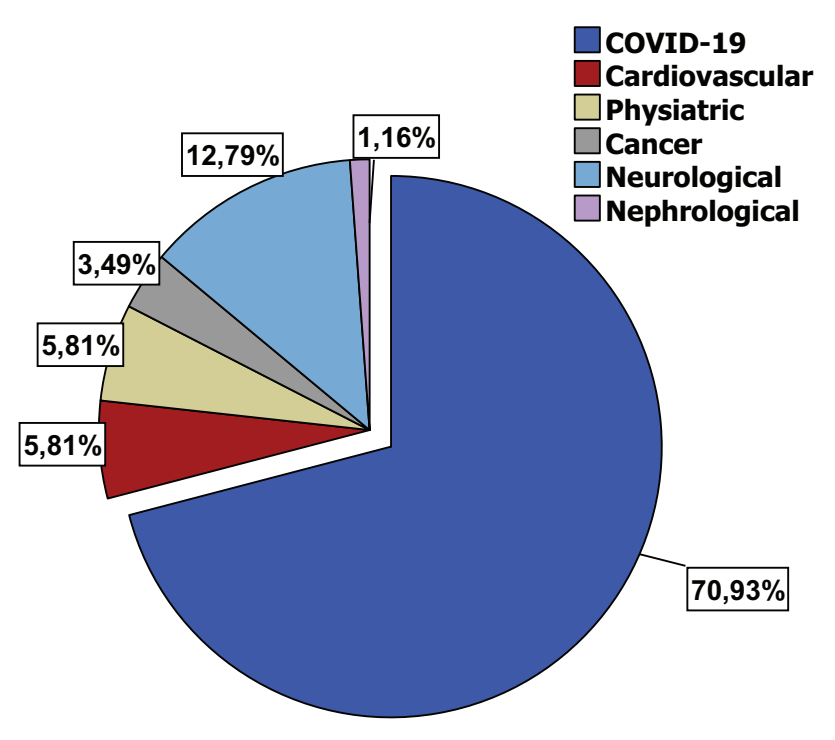

Figure 2 Medical diagnoses of participants at admission.

Notes: These are the medical diagnoses of participants at the admission to the rehabilitation Institute. COVID 19 = patients only with COVID-19 disease; other = patients with other diagnoses beyond COVID-19 disease.

a new diagnosis of cognitive impairment $(n=4,4.7 \%)$, acute stress disorders $(\mathrm{n}=16,18.6 \%)$, anxiety states and/or demoralization symptoms $(\mathrm{n}=23,26.7 \%)$, depression $(\mathrm{n}=9,10.5 \% \%)$, and troublesome grief $(n=7,8.1 \%)$. Once recovered from the COVID-19, 33 patients (38.4\%) were discharged, returning home or in a nursing home, and $36(41.9 \%)$ continued the rehabilitation in non-COVID-19 departments. 26 (72.2\%) of the latter continued because of pre-existent medical issues and $10(27.8 \%)$ because of complications from COVID-19.

Aside from those with cognitive impairment $(n=6), 30$ patients of the previously treated and no more positive for COVID-19, overcame the most critical psychological phase, but still needed psychological support. This was continued also in non-COVID-19 rehabilitation wards, with the aim to manage residual anxiety and fear - possible Post Traumatic Stress Disorder (PTSD), improve coping skills, and/or reschedule their life (especially for recently widowed patients).

At the end of data collection, 9 patients died (10.5\%) and $7(8.1 \%)$ were still hospitalized in the COVID-19 ward.

Although the data on caregivers was not collected, 60 of them received telephonic psychological support to cope with the situation. 


\section{Discussion}

This study focused on the psychological issues and difficulties experienced by patients with post-acute COVID-19 who were admitted to rehabilitation, with particular attention to the psychological intervention offered them according to a CBT framework. Indeed, since the beginning, patients' need for professional psychological help has emerged in its importance.

According to results, $47.5 \%(n=86)$ of patients in the COVID-19 ward underwent psychological assessment. Among them, 7\% showed good adaptation, $11.6 \%$ were supported only with video calls, whilst a CBT psychological treatment was fundamental for $75.59 \%$ of them.

Considering all the patients with COVID-19 ( $n=181)$, those who required structured psychological treatment were $35.91 \%(\mathrm{n}=65)$.

The CBT approach was used; CBT is evidence-based psychotherapy widely used to prevent and reduce psychophysical distress in the general population ${ }^{38}$ and among inpatients as well. ${ }^{39}$ According to the literature, $\mathrm{CBT}$ is the most effective and efficient psychotherapy approach to relieve psychological distress and related symptoms, such as insomnia ${ }^{40}$ and physical fatigue, ${ }^{41,42}$ even when short programs are used. Regardless of the specific symptomatology, the CBT intervention aimed to help inpatients to identify psychological stress levels and to modify their dysfunctional cognitive beliefs, emotions, and behaviors, ${ }^{33}$ thus reduce symptomatology and psychological sufferance. To do so, various techniques can be used cognitive reconstruction, behavioral modification, social support - and can be adapted to each individual. Inpatients were also helped to restore their psychological and social functioning to go back to daily life, thus improving quality of life and well-being.

The COVID-19 patients showed particular psychological issues related to their current physical condition and the ongoing pandemic.

Acute stress disorders were common among patients ( $\mathrm{n}=16,18.6 \%$ ) showing all the typical symptoms, such as insomnia, nightmares, rumination, intrusive thoughts. Indeed, patients with COVID-19 were facing a stressful and prolonged situation seriously compromising their health. Also, most patients lived traumatic experiences, such as the intense fear of dying due to medical issues (eg, severe respiratory difficulties), or saw in first person people dying of COVID-19 complications, such as relatives or fellow patients at the former hospital they were treated at. The psychological impact of life-threatening experiences is considerable and can be long lasting if not treated. Considering these stressful and traumatic experiences, in the psychological intervention the Eye Movement Desensitization and Reprocessing therapy was among the election choices to treat these symptoms and the associated psychological sufferance. ${ }^{43}$

Troublesome grieving was reported by $8.1 \%(\mathrm{n}=7)$ of patients who lost their spouse. As literature shows, during the COVID-19 pandemic grief was particularly difficult due to the impossibility to see the deceased spouse and say goodbye for the last time. Besides, the funeral ban did not allow any memorial ceremony. ${ }^{44}$ Some of these patients lost the spouse just before hospitalization or were informed of the death during their hospital stay $(n=5)$. In the latter case, psychologists favored the communication of the loss from the caregivers to the patient via a video call with a digital tablet. All these conditions made patients incredulous about the spouse's loss and made even more difficult the grieving process. Furthermore, after experiencing the death of their loved ones, some patients reported intense fear of their own death, despite signs of physical recovery. The psychological intervention, beyond using EMDR techniques, focused on coping with the bereavement that, according to the Dual Process Model, ${ }^{45}$ can be promoted by favoring the restoration oriented experiences rather than the loss oriented ones.

Anxiety states and demoralization symptoms were displayed by $26.7 \%(n=23)$ of these patients, negative mood disturbances were characterized by sadness and anxious feelings related both to the current situation and the future, perceived as confused and uncertain. Guilt reactions and worries emerged for the fear of having infected other family members. Furthermore, feelings of shame and stigmatization were common among patients with COVID$19,{ }^{46}$ especially for those who suffered severe physical damage (eg, amputation) and who had difficulties in figuring how family, friends, and colleagues would react.

A more severe depressive syndrome affected 10.5\% (n $=9$ ) of patients. This condition had relevant severity and was often associated with the previous individual history and/or with complex consequences of COVID-19, such as severe neuropathy or tracheotomy. One patient also reported suicidal thoughts and was then strictly monitored by health-professionals.

The psychological treatment for anxious and depressive issues mainly consisted of classic CBT techniques for 
anxiety and depression, ${ }^{47}$ specific techniques to increase the tolerance of uncertainty, ${ }^{48}$ as well as novel approaches as the Schema Therapy allowing to address symptomatology that is deeply rooted in the individual life history. ${ }^{49-51}$

The patients with cognitive impairment $(n=22,25.6 \%)$ showed more severe disorientation with some manifestations of intense fear and panic (eg, trembling, screaming) at the sight of health professionals in unfamiliar protective suits. For them, the psychological intervention consisted in the containment of panic reactions and in emotional reassurance.

Regarding the rehabilitation outcomes, although the patients transferred to non-COVID-19 wards improved their psychological condition according to the clinicians' judgment, psychological treatment was continued because they still exhibited residual difficulties (possible PTSD) still requiring treatment. The patients discharged home declared to be satisfied with the psychological intervention and some needed to continue psychological counseling in their territorial services.

Even though caregivers were not included in the study, their psychological conditions deserve clinical attention. ${ }^{52}$ They were stressed by the patient hospitalization and because of the forced physical separation and they showed difficulties in understanding the situation and their beloved ones' experience. Despite the great desire to see each other again, they showed fear to cause additional problems. Furthermore, the sudden unemployment or the decision to resign, as a consequence of the pandemic, inevitably resulted in worrying financial situations for some family members. A total of 60 caregivers received telephonic psychological support. Among them, anxiety, fear, worry, uncertainty about the future, mood swings, weeping, sleep disturbances, sadness, and guilt (eg: for infecting the patient) were often reported.

One important limitation of this study was the impossibility to retrieve psychological pre-post data about patients by using formalized assessment tools; in this exceptional emergency was preferred to conduct the assessment with the clinical interview in order to maximize the time-resources ratio and to allow frightened patients to feel welcomed. Consequently, it was not possible to quantify the psychological improvement and the effect of psychological intervention during the rehabilitation stay with the use of standardized questionnaires but only with the clinical judgment. ${ }^{53}$ Moreover, the study was focused on patients, and data about caregivers was not collected.
Future research should explore the psychological reactions of COVID-19 patients and the related psychological interventions in order to compare these findings with those of other hospitals, patients of different ages, and other countries. Another interesting approach for future research is the use of thematic analysis (eg, narrative) to deepen the subjective meaning of the psychological experience of each patient. ${ }^{54}$ Thus, regardless of different conditions and resources, the uniqueness of COVID-19 psychological impact should be highlighted in comparison to other epidemics and illnesses that did not reach these devastating consequences.

As a strength, this study was performed by psychologists who personally worked inside a rehabilitation department for post-acute COVID-19 patients. The findings of this study may be extended to other critical contexts where COVID-19 or other severe infectious diseases are implied.

Finally, it is important to underline that, given the kind of psychological issues, COVID-19 patients and their caregivers could experience various psychological issues in hospital as well as in a home care environment despite these circumstances pose different challenges. Indeed, despite the psychological impact of the COVID-19 may be long-lasting, once at home people may not feel comfortable with seeking psychological help ${ }^{55,56}$ because of the stigma associated with psychological and psychiatric issues $^{57-60}$ thus resulting in a large number of people that do not receive treatment for emotional problems. Thus, once discharged from the hospital/rehabilitation facility patients and caregivers need to be followed up by implementing web-based and/or vis a vis psychological support and interventions in order to monitor and promote their health over time. ${ }^{61-63}$

\section{Conclusion}

In conclusion, this study showed that patients affected by COVID-19 evidenced psychological difficulties that deserve careful clinical attention, moreover, the psychological needs of patients with COVID-19 (and their caregivers) should be identified and treated. It is important to understand their emotional difficulties and to promote psychological health with tailored psychological interventions. Despite the constraints encountered during the COVID-19 pandemic, it was possible to adapt the psychological intervention also in such a particular context in order to face the emergency. However, more specific measures will be needed to bring the COVID-19 emergency intervention to the quality of normal interventions. 


\section{Acknowledgments}

We are sincerely grateful to Diana Sacco for language editing.

\section{Author Contributions}

All authors made a significant contribution to the work reported, whether that is in the conception, study design, execution, acquisition of data, analysis and interpretation, or in all these areas; took part in drafting, revising or critically reviewing the article; gave final approval of the version to be published; have agreed on the journal to which the article has been submitted; and agree to be accountable for all aspects of the work.

\section{Disclosure}

The authors report no conflicts of interest in this work.

\section{References}

1. Zaim S, Chong JH, Sankaranarayanan V, Harky A. COVID-19 and multi-organ response. Curr Probl Cardiol. 2020;45(8):100618. doi:10.1016/j.cpcardiol.2020.100618

2. Imai N, Cori A, Dorigatti I, et al. Report 3: transmissibility of 2019-nCoV. Imp Coll London. 2020. doi:10.25561/77148

3. Istituto Superiore di Sanità. Integrated surveillance of COVID-19 in Italy; 2020. Available from: https://www.epicentro.iss.it/en/coronavirus/ bollettino/Infografica_13maggioENG.pdf. Accessed May 14, 2020.

4. Cerami C, Santi GC, Galandra C, et al. COVID-19 outbreak in Italy: are we ready for the psychosocial and economic crisis? Baseline findings from the longitudinal psyCovid study. SSRN Electron J. 2020. doi: $10.2139 / \mathrm{ssrn} .3569868$

5. Favieri F, Forte G, Tambelli R, Casagrande M. The Italians in the time of coronavirus: psychosocial aspects of unexpected COVID-19 pandemic. SSRN Electron J. 2020. doi:10.2139/ssrn.3576804

6. Lai J, Ma S, Wang Y, et al. Factors associated with mental health outcomes among health care workers exposed to coronavirus disease 2019. JAMA Netw open. 2020;3(3):e203976. doi:10.1001/ jamanetworkopen.2020.3976

7. Nese M, Riboli G, Brighetti G, et al. Delay discounting of compliance with containment measures during the COVID-19 outbreak: a survey of the Italian population. J Public Health. 2020. doi:10.1007/s10389-020-01317-9

8. Qiu J, Shen B, Zhao M, Wang Z, Xie B, Xu Y. A nationwide survey of psychological distress among Chinese people in the COVID-19 epidemic: implications and policy recommendations. Gen Psychiatry. 2020;33(2):19-21. doi:10.1136/gpsych-2020-100213

9. Rossi A, Panzeri A, Pietrabissa G, Manzoni GM, Castelnuovo G, Mannarini S. The anxiety-buffer hypothesis in the time of Covid-19: when self-esteem protects from the impact of loneliness and fear on anxiety and depression. Front Psychol. 2020;11. doi:10.3389/ fpsyg.2020.02177.

10. Chen Q, Liang M, Li Y, et al. Mental health care for medical staff in China during the COVID-19 outbreak. Lancet Psychiatry. 2020;7(4): e15-e16. doi:10.1016/S2215-0366(20)30078-X

11. Wang C, Pan R, Wan X, et al. Immediate psychological responses and associated factors during the initial stage of the 2019 coronavirus disease (COVID-19) epidemic among the general population in China. Int J Environ Res Public Health. 2020;17:5. doi:10.3390/ ijerph17051729
12. Zhou X. Psychological crisis interventions in Sichuan Province during the 2019 novel coronavirus outbreak. Psychiatry Res. 2020;286 (February):112895. doi:10.1016/j.psychres.2020.112895

13. Huremović D. Psychiatry of Pandemics. A Mental Health Response to Infection Outbreak. Huremović D, editor. Springer International Publishing; 2019. doi:10.1007/978-3-030-15346-5

14. Parola A, Rossi A, Tessitore F, Troisi G, Mannarini S. Mental health through the COVID-19 quarantine: a growth curve analysis on Italian young adults. Front Psychol. 2020;11. doi:10.3389/ fpsyg. 2020.567484

15. Armitage R, Nellums LB. COVID-19 and the consequences of isolating the elderly. Lancet Public Health. 2020;5(5):e256. doi:10.1016/S2468-2667(20)30061-X

16. de Girolamo G, Cerveri G, Clerici M, et al. Mental health in the coronavirus disease 2019 emergency-The Italian Response. JAMA Psychiatry. 2020;77(9):974. doi:10.1001/jamapsychiatry.2020.1276

17. CNOP. Digitalizzazione Della Professione e Dell'intervento Psicologico Mediato Dal Web. Rome; 2017. Available from: https:// d66rp9rxjwtwy.cloudfront.net/wp-content/uploads/2015/04/AttiTipici DEF interno-LR-1.pdf. Accessed May 14, 2020.

18. Consiglio Nazionale Ordine degli Psicologi. Linee Di Indirizzo per l'intervento Psicologico a Distanza a Favore Della Popolazione Nell'emergenza Covid-19. Roma; 2020. Available from: https:// d66rp9rxjwtwy.cloudfront.net/wp-content/uploads/2020/04/LINEEDI-INDIRIZZO-PER-LINTERVENTO-PSICOLOGICO -A-DISTANZA-A-FAVORE-DELLA-POPOLAZIONENELLEMERGENZA-COVID-19-1.pdf. Accessed May 14, 2020.

19. Orrù $\mathrm{G}$, Ciacchini R, Gemignani $\mathrm{A}$, Conversano $\mathrm{C}$. Psychological intervention measures during the COVID-19 pandemic. Clin Neuropsychiatry. 2020;17(2):76-79. doi:10.36131/CN20200208

20. Panzeri A, Rossi Ferrario S, Vidotto G. Interventions for psychological health of stroke caregivers: a systematic review. Front Psychol. 2019;10(Article2045):1-16. doi:10.3389/fpsyg.2019.02045

21. Ratti MM, Rossi A, Delli Zotti GB, Sarno L, Spotti D. Social support, psychological distress and depression in hemodialysis patients. Psicol della Salut. 2017;(1):112-122. doi:10.3280/ PDS2017-001006

22. Balestroni G, Panzeri A, Omarini P, et al. Psychophysical health of elderly inpatients in cardiac rehabilitation: a retrospective cohort study. Eur J Phys Rehabil Med. 2020;56(2):2. doi:10.23736/S19739087.20.05970-5

23. Rossi Ferrario S, Panzeri A. Exploring illness denial of LVAD patients in cardiac rehabilitation and their caregivers: a preliminary study. Artif Organs. 2020;44(6):655-660. doi:10.1111/aor.13630

24. Mannarini S. A method for the definition of a self-awareness behavior dimension with clinical subjects: a latent trait analysis. Behav Res Methods. 2009;41(4):1029-1037. doi:10.3758/BRM.41.4.1029

25. Ferrario SR, Panzeri A, Anselmi P, Vidotto G. Development and psychometric properties of a short form of the illness denial questionnaire. Psychol Res Behav Manag. 2019;12. doi:10.2147/ PRBM.S207622.

26. Pietrabissa G, Manzoni GM, Rossi A, Castelnuovo CG. The MOTIV-HEART study: a prospective, randomized, single-blind pilot study of brief strategic therapy and motivational interviewing among cardiac rehabilitation patients. Front Psychol. 2017;8:1-12. doi: $10.3389 /$ fpsyg. 2017.00083

27. Pietrabissa G, Sorgente A, Rossi A, et al. Stages of change in obesity and weight management: factorial structure of the Italian version of the University of rhode island change assessment scale. Eat Weight Disord. 2017;22(2):361-367. doi:10.1007/s40519-016-0289-1

28. Cattivelli R, Castelnuovo G, Musetti A, et al. ACTonHEALTH study protocol: promoting psychological flexibility with activity tracker and mHealth tools to foster healthful lifestyle for obesity and other chronic health conditions. Trials. 2018;19(1):1. doi:10.1186/s13063018-2968-x 
29. Rossi Ferrario S, Omarini P, Cerutti P, Balestroni G, Omarini G, Pistono PM. When LVAD patients die: the Caregiver's mourning Artif Organs. 2016;40(5):454-458. doi:10.1111/aor.12594

30. Bennett KM. How to achieve resilience as an older widower: turning points or gradual change? Ageing Soc. 2010;30(3):369-382. doi:10.1017/S0144686X09990572

31. Moen T. Reflections on the narrative research approach. Int J Qual Methods. 2006;5(4):56-69. doi:10.1177/160940690600500405

32. American Psychiatric Association. Diagnostic and Statistical Manual of Mental Disorders. 5th Ed. Washington, DC; 2013.

33. Beck AT. Cognitive Therapy and the Emotional Disorders. New York, NY: Penguin; 1979.

34. Cayoun B. Mindfulness-Integrated CBT: Principles and Practice. John Wiley \& Sons; 2011.

35. Panzeri A, Rossi Ferrario S. Supporting rehabilitation patients with COVID-19 during the pandemic: experiences from a technologybased psychological approach. In: Gigliotta O, Ponticorvo M, Eds. CEUR Workshop Proceedings: SecondSymposium on PsychologyBased Technologies - Psychobit Vol. 2730. Naples; 2020.

36. R Core Team. R: a language and environment for statistical computing. R Foundation for Statistical Computing:Vienna, Austria. Available from: http://www.R-project.org/. Accessed January 27, 2021..

37. Association WM. World medical association declaration of Helsinki: ethical principles for medical research involving human subjects. JAMA. 2013;310(20):2191-2194. doi:10.1001/jama.2013.281053.

38. Schure MB, Lindow JC, Greist JH, et al. Use of a fully automated internet-based cognitive behavior therapy intervention in a community population of adults with depression symptoms: randomized controlled trial. J Med Internet Res. 2019;21(11):11. doi: $10.2196 / 14754$

39. Ólason M, Andrason RH, Jónsdóttir IH, Kristbergsdóttir H, Jensen MP. Cognitive behavioral therapy for depression and anxiety in an interdisciplinary rehabilitation program for chronic pain: a randomized controlled trial with a 3-year follow-up. Int J Behav Med. 2018;25(1):55-66. doi:10.1007/s12529-017-9690-z

40. Ballesio A, Aquino MRJ V, Feige B, et al. The effectiveness of behavioural and cognitive behavioural therapies for insomnia on depressive and fatigue symptoms: a systematic review and network meta-analysis. Sleep Med Rev. 2018;37:114-129. doi:10.1016/j. smrv.2017.01.006

41. Manzoni GM, Rossi A, Marazzi N, et al. Feasibility, validity, and reliability of the Italian pediatric quality of life inventory multidimensional fatigue scale for adults in inpatients. Obes Facts. 2018;11 (1):25-36. doi:10.1159/000484565

42. Yang YL, Sui GY, Liu GC, Huang DS, Wang SM, Wang L. The effects of psychological interventions on depression and anxiety among Chinese adults with cancer: a meta-analysis of randomized controlled studies. BMC Cancer. 2014;14(1):956. doi:10.1186/14712407-14-956

43. Solomon RM, Hensley BJ, Therapy EMDR. Treatment of grief and mourning in times of COVID-19 (Coronavirus). $J$ EMDR Pract Res. 2020;14(3):162-174. doi:10.1891/EMDR-D-20-00031

44. Carr D, Boerner K, Moorman S. Bereavement in the time of coronavirus: unprecedented challenges demand novel interventions. $J$ Aging Soc Policy. 2020;32(4-5):425-431. doi:10.1080/08959420.20 20.1764320

45. Stroebe M, Schut H. Bereavement in times of COVID-19: a review and theoretical framework. Omega. 2021;82(3):500-522. doi:10.1177/0030222820966928

46. Mannarini S, Rossi A. Assessing mental illness stigma: a complex issue. Front Psychol. 2019;9. doi:10.3389/fpsyg.2018.02722.

47. Li J, Li X, Jiang J, et al. The effect of cognitive behavioral therapy on depression, anxiety, and stress in patients with COVID-19: a randomized controlled trial. Front Psychiatry. 2020;11:580827. doi:10.3389/fpsyt.2020.580827
48. Robichaud M, Dugas MJ. A cognitive-behavioral treatment targeting intolerance of uncertainty. In: Davey GCL, Wells A, Robichaud M, Dugas MJ, editors. G. C. L. DaveyWorry and Its Psychological Disorders: Theory, Assessment and Treatment. Chichester, UK: Wiley; 2006.289-304.

49. Hawke LD, Provencher MD. Schema theory and schema therapy in mood and anxiety disorders: a review. Artic $J$ Cogn Psychother. 2011;25(4):257-276. doi:10.1891/0889-8391.25.4.257

50. Pietrabissa G, Rossi A, Simpson S, et al. Evaluation of the reliability and validity of the Italian version of the schema mode inventory for eating disorders: short form for adults with dysfunctional eating behaviors. Eat Weight Disord. 2020;25(3):553-565. doi:10.1007/ s40519-019-00644-5

51. Simpson SG, Pietrabissa G, Rossi A, et al. Factorial structure and preliminary validation of the Schema Mode Inventory for Eating Disorders (SMI-ED). Front Psychol. 2018;9:(APR):1-17. doi:10.3389/fpsyg.2018.00600

52. Kent EE, Ornstein KA, Dionne-Odom JN. The family caregiving crisis meets an actual pandemic. J Pain Symptom Manage. 2020;60 (1):e66-e69. doi:10.1016/j.jpainsymman.2020.04.006

53. Turner D, Schünemann HJ, Griffith LE, et al. The minimal detectable change cannot reliably replace the minimal important difference. $J$ Clin Epidemiol. 2010;63(1):28-36. doi:10.1016/j.jclinepi.2009.01.024

54. Parola A. Novel coronavirus outbreak and career development: a narrative approach into the meaning of Italian university graduates. Front Psychol. 2020;11:2255. doi:10.3389/FPSYG.2020.02255

55. Rossi A, Mannarini S. The Italian version of the attitudes toward seeking professional psychological help scale - short form: the first contribution to measurement invariance. TPM Test Psychom Methodol Appl Psychol. 2019;26(1):93-100. doi:10.4473/TPM26.1.5

56. Mannarini S, Boffo M, Balottin L. Beliefs about the patient's role in the psychotherapeutic relationship: a latent trait perspective. TPM Test Psychom Methodol Appl Psychol. 2013;20:277-294. doi:10.4473/tpm20.3.6

57. Mannarini S, Rossi A, Munari C. How do education and experience with mental illness interact with causal beliefs, eligible treatments and stigmatising attitudes towards schizophrenia? A comparison between mental health professionals, psychology students, relatives and patients. $B M C$ Psychiatry. 2020;20(1):11. doi:10.1186/s12888-020-02580-6

58. Mannarini S, Boffo M, Rossi A, Balottin L. Etiological beliefs, treatments, stigmatizing attitudes towards schizophrenia. What do Italians and Israeli think? Front Psychol. 2018;8(2289):1-9. doi:10.3389/fpsyg.2017.02289

59. Mannarini S, Boffo M, Urgesi C. An implicit measure of associations with mental illness versus physical illness: response latency decomposition and stimuli differential functioning in relation to IAT order of associative conditions and accuracy. PLoS One. 2014;9(7):101911. doi:10.1371/journal.pone.0101911

60. Mannarini S, Boffo M. Assessing mental disorder causal beliefs: a latent dimension identification. Community Ment Heal J. 2013;49 (6):686-693. doi:10.1007/s10597-012-9581-3

61. Zhou X, Snoswell CL, Harding LE, et al. The role of telehealth in reducing the mental health burden from COVID-19. Telemed $J$ E Health. 2020;26(4):26-28. doi:10.1089/tmj.2020.0068

62. Duan L, Zhu G. Psychological interventions for people affected by the COVID-19 epidemic. Lancet Psychiatry. 2020;7(4):300-302. doi:10.1016/S2215-0366(20)30073-0

63. Jackson JB, Pietrabissa G, Rossi A, Manzoni GM, Castelnuovo G. Brief strategic therapy and cognitive behavioral therapy for women with binge eating disorder and comorbid obesity: a randomized clinical trial one-year follow-up. J Consult Clin Psychol. 2018;86 (8):688-701. doi:10.1037/ccp0000313 


\section{Publish your work in this journal}

Neuropsychiatric Disease and Treatment is an international, peerreviewed journal of clinical therapeutics and pharmacology focusing on concise rapid reporting of clinical or pre-clinical studies on a range of neuropsychiatric and neurological disorders. This journal is indexed on PubMed Central, the 'PsycINFO' database and CAS, and is the official journal of The International Neuropsychiatric Association (INA). The manuscript management system is completely online and includes a very quick and fair peer-review system, which is all easy to use. Visit http://www.dovepress.com/testimonials.php to read real quotes from published authors.

Submit your manuscript here: https://www.dovepress.com/neuropsychiatric-disease-and-treatment-journal 